\title{
Fabrication of Highly Ordered Multilayer Thin Films and Its Applications
}

\author{
Jinhan Cho, Sangcheol Kim and Kookheon Char \\ School of Chemical Engineering, Seoul National University, San 56-1, Shinlim-dong, Kwanak-gu, Seoul 151-744, Korea \\ (Received 20 June $2002 \cdot$ accepted 8 August 2002)
}

\begin{abstract}
A new method is introduced to build up organic/organic multilayer films composed of cationic poly(allylamine hydrochloride) (PAH) and negatively charged poly(sodium 4-styrenesulfonate) (PSS) by using the spinning process. The adsorption process is governed by both the viscous force induced by fast solvent elimination and the electrostatic interaction between oppositely charged species. On the other hand, the centrifugal and air shear forces applied by the spinning process significantly enhance the desorption of weakly bound polyelectrolyte chains and also induce the planarization of the adsorbed polyelectrolyte layer. The film thickness per bilayer adsorbed by the conventional dipping process and the spinning process was found to be about $4 \AA$ and $24 \AA$, respectively. The surface of the multilayer films prepared with the spinning process is quite homogeneous and smooth. Electroluminescence (EL) devices composed of alternating poly( $p$-phenylene vinylene) (PPV) and polyanions films show higher quantum efficiency when prepared by the spin self-assembly (SA) method.
\end{abstract}

Key words: Spin Self-Assembly Method, Multilayer, Electrostatic Attraction, Electroluminescence Device

\section{INTRODUCTION}

Fabrication of multilayer thin films has attracted much interest because they are of great use in thin film devices based on electrochemical processes such as sensors, integrated optics, rectifiers, or light-emitting devices (LED) [Ho et al., 2000; Zhang et al., 1999; Mendelson et al., 2000; Park et al., 2001; Kim et al., 1997; Laschewasky et al., 1997; He et al., 1999]. Most of these applications require the preparation of stable and well-organized films with fast fabrication processes. Since the ionic layer-by-layer self-assembly (SA) technique was introduced for the fabrication of polyelectrolyte multilayer, this self-assembling technique has been extended to conducting polymer composites, nonlinear optical dyes, and the assembly of nanoparticles or biomolecular systems [Decher, 1997; Lenahan et al., 1998; Kotov et al., 1995; Lvov et al., 1995]. This layer-bylayer SA method is principally based on self-diffusion rearrangement, and thus various experimental factors should be taken into account in order to increase the surface coverage of a polymer layer adsorbing onto a substrate: the adsorption time, the $\mathrm{pH}$ control, the concentration of polyelectrolyte and the amount of added ionic salt. After the adsorption of a polyelectrolyte layer, thorough washing using a flow of a pure solvent is required because the weakly adsorbed polyelectrolyte chains significantly increase the surface roughness of the multilayer films.

According to numerous studies on the polymer LEDs, it is known that the polymer/electrode interfaces play important roles in determining the operating characteristics and the stability of the devices [Ho et al., 1998; Onitsuka et al., 1996]. Recently, the layer-by-layer SA method based on the electrostatic attraction between opposite charges has been implemented in LED fabrication. It was demonstrated that the film thickness could be easily controlled at the molecular level and the desired heterogeneous layers could be inserted

To whom correspondence should be addressed.

E-mail:khchar@plaza.snu.ac.kr in the organic thin film structure [Hong et al., 1998]. It also contributes to the better understanding of the roles of the heterogeneous layers for the charge injection at the interfaces between organic layers and electrodes as well as for the effect of film thickness on the recombination zone (i.e., film thickness for polaron excitons created through the recombination of holes and electrons).

In this work, we describe the new spin self-assembly (SA) method as an alternative for producing well-organized multilayer films in short process time. Poly(allylamine hydrochloride) (PAH) and poly(sodium 4-styrenesulfonate) (PSS) were used as a cationic and an anionic polymer, respectively. Through the analyses by UV-Vis spectroscopy, AFM and contact angle measurement, the multilayer thin films fabricated by the spin SA method were compared with those prepared by the conventional dipping method. We have also investigated the operating characteristics of electroluminescence (EL) devices composed of alternating poly (p-phenylene vinylene) (PPV) and polyanions such as PSS and poly(methacrylic acid) (PMA) films. These multilayer devices were prepared by using the conventional layer-by-layer SA method and the spin SA method.

\section{EXPERIMENTAL}

\section{Materials}

Poly(sodium 4-styrenesulfonate) (PSS) (Aldrich, $\mathrm{M}_{W}=70,000$ ) and poly(methacrylic acid) (PMA) (Polyscience, $\left.\mathrm{M}_{W}=15,000\right)$ were used as anionic polyelectrolytes. As a cationic polymer, poly(allylamine hydrochloride) (PAH) (Aldrich, $\left.\mathrm{M}_{n}=50,000-65,000\right)$ was used. All the polyelectrolyte aqueous solutions were used without $\mathrm{pH}$ adjustment and the ionic strength of the PSS solution was adjusted by the addition of $\mathrm{NaCl}$. PPV precursor was prepared by the polymerization of a bis-sulphonium salt of $p$-xylene using the method given by Lenz et al. The reaction product was dialyzed with a molecular weight cut-off at 12,000 and the concentration of PPV precursor in aqueous solution was estimated by gravimetry to be $0.013 \mathrm{M}$. 


\section{Multilayer Preparation}

Quartz substrates for the deposition of polyelectrolytes were initially cleaned by ultrasonification in a hot mixture of $\mathrm{H}_{2} \mathrm{SO}_{4} / \mathrm{H}_{2} \mathrm{O}_{2}$ $(7 / 3)$ for 3 hours. They were then heated in a mixture of $\mathrm{H}_{2} \mathrm{O} / \mathrm{H}_{2} \mathrm{O}_{2} /$ $\mathrm{NH}_{3}(5 / 1 / 1)$ at $80^{\circ} \mathrm{C}$ for 1 hour, and then subsequently dried by $\mathrm{N}_{2}$ gas purging. After this procedure, the substrates were negatively charged and used for the polyelectrolyte deposition starting with the cationic PAH. In the conventional layer-by-layer SA method, the substrates were immersed alternately in the cationic solution and in the anionic solution for $20 \mathrm{~min}$ for each deposition. After each deposition step, the surface of the self-assembled film was washed by dipping in deionized water for 2 min and then blown dry with a stream of nitrogen. In the spin SA method, the deposition steps were carried out as follows: a few drops of polyelectrolyte solution were placed on the substrate and then the substrate was rotated with a spinner at a fixed rotating speed (typically, 4,000 rpm) for 8 to 15 seconds. After the deposition of each polyelectrolyte layer, the substrates were thoroughly rinsed twice with plenty of deionized water. The spinning time and the speed for the washing step were also identical to those for the layer deposition. For the preparation of EL devices, the films were then heated to $230^{\circ} \mathrm{C}$ for 1 hour under vacuum for the PPV conversion after the multilayer deposition.

\section{Characterization}

The characteristics of multilayer films were analyzed by UV-Vis spectrometer, atomic force microscope (AFM) in contact mode and contact angle measurement. An ellipsometer was also employed for the thickness measurement.

Polymer LEDs based on the self-assembly were prepared on indium tin oxide (ITO) coated glasses. The thickness of the ITO layer is approximately $1,850 \AA$ and the sheet resistance is $10-20 \Omega \mathrm{cm}$. The ITO substrates were cleaned by sonication in isopropyl alcohol for 1 hour, followed by immersion in a mixture of $\mathrm{H}_{2} \mathrm{O} / \mathrm{H}_{2} \mathrm{O}_{2} /$ $\mathrm{NH}_{3}$ (5/1/1) for 1 hour. After the thermal conversion of the PPV precursor to a conjugated form of PPV, aluminium counter electrode was evaporated onto the multilayer films. The active area for light emission is $16 \mathrm{~mm}^{2}$. The current-voltage-light (I-V-L) charac-

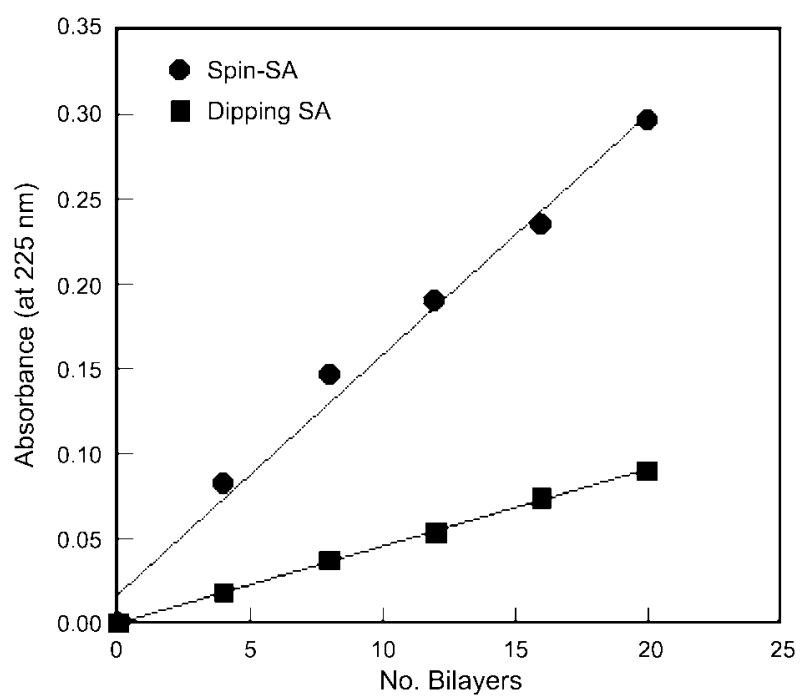

Fig. 1. Absorbance at $225 \mathrm{~nm}$ of PAH/PSS multilayers prepared by (a) spin SA method and (b) dipping SA method. teristics were recorded on a Keithly 236 source/measure unit for the V-I relationship and by a Newport SIS-SL photodiode for the V-L intensity correlation.

\section{RESULTS AND DISCUSSION}

Fig. 1 shows the difference in UV-Vis absorbance of multilayer
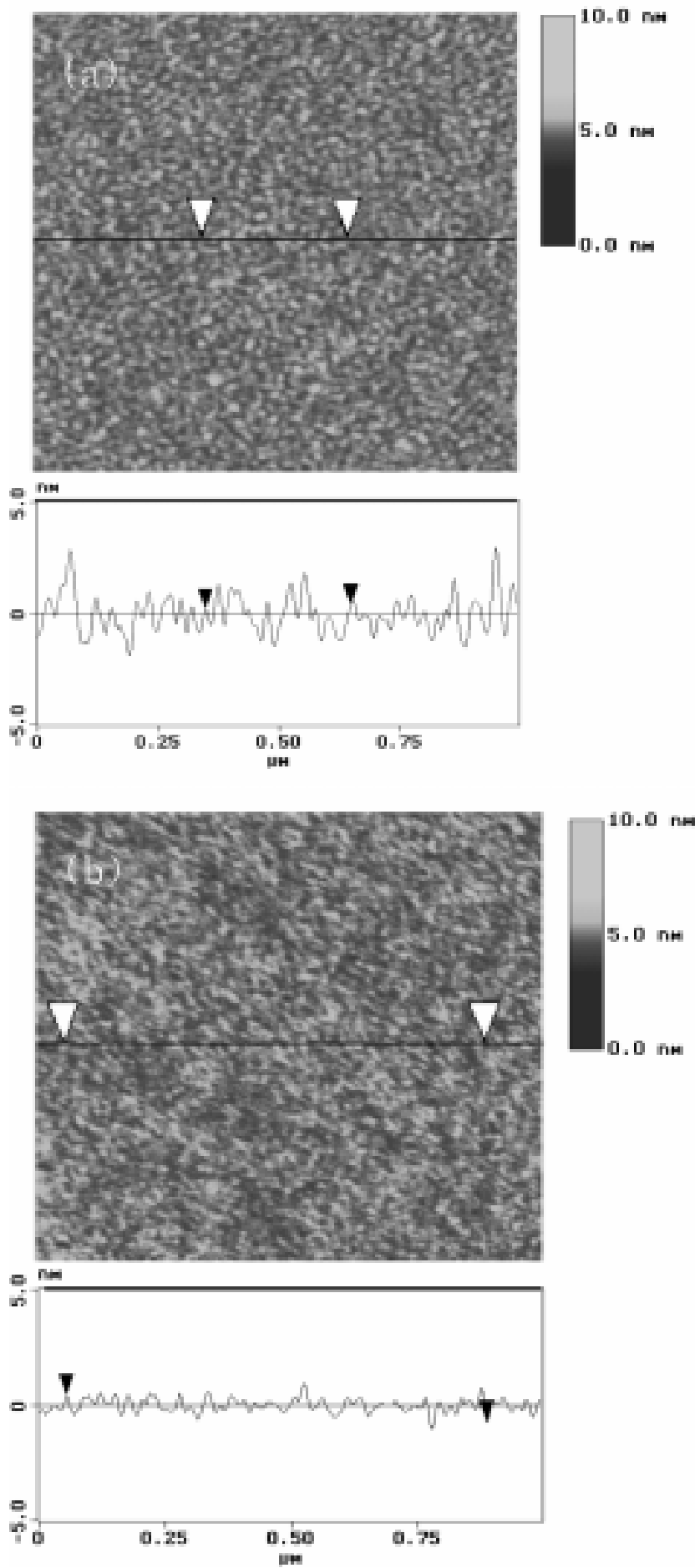

Fig. 2. (a) Tapping mode AFM images (Digital Instruments, Nanoscope IIIa) of a (PAH/PSS $)_{30}$ film prepared with the dipping SA and (b) a (PAH/PSS $)_{30}$ film fabricated with the spin SA. 
films as a function of bilayer number prepared by both spin SA and dipping SA methods. Due to the contribution of the adsorbed PSS chains, the absorbance occurs at $225 \mathrm{~nm}$ in the UV region. The aqueous polyelectrolyte concentrations of PAH and PSS used in the two different deposition methods were all identical to be $10 \mathrm{mM}$. The dipping process was performed for an adsorption time of $20 \mathrm{~min}$ per layer in order to allow enough time for the saturated adsorption. In spin SA process, however, the amount of polymers adsorbed at each deposition step was found to be much larger. It should be noted at this point that the multilayer film fabricated by the spin SA method is adsorbed onto only one side of a quartz wafer, while the film prepared by the dipping SA method is adsorbed onto both sides of a quartz wafer. The film thickness per bilayer adsorbed by the dipping process and the spinning process was found to be about $4 \AA$ and $24 \AA$, respectively, as determined from the ellipsometric measurement.

We also found that the spin SA method has a significant effect on the surface roughness of prepared multilayer films presumably due to the air shear force during the spinning process [Chou et al., 2000]. In order to investigate this effect in detail, we measured the surface roughness of the self-assembled films using atomic force microscopy (AFM) in tapping mode as shown in Fig. 2. Although the flattened adsorption of the polyelectrolyte onto a substrate was induced by low ionic strength, the surface roughness of the dipping SA film composed of (PAH/PSS) $)_{30}$ multilayer yield $8.1 \AA$ An One other hand, the (PAH/PSS) multilayer film prepared by the spin SA method shows a surface roughness of about 3.1 $\mathrm{A}$. These results are quite reproducible and clearly demonstrate that the air shear force driven by the spinning process significantly enhances the surface planarization of the multilayer films. In addition, the surface roughness of about $3.1 \AA$ and the bilayer thickness of $24 \AA$ for the spinning process compared with $8.1 \AA$ and $4 \AA$ of equivalent properties for the dipping process provides an indirect evidence that the internal structure of the spin SA films is highly ordered. This also points to the fact that the film prepared with the spin process contains rather distinct interfaces between respective layers in contrast to the film obtained by the dipping method, although the Bragg peaks in the $\mathrm{X}$-ray reflectivity spectra of the PAH/PSS multilayer films cannot distinguish such internal structure due to small electron density difference between PAH and PSS.

The significant difference in the adsorbed amount and the surface roughness between the dipping and the spinning method is caused by the different adsorption mechanism. The conventional SA method by the dipping process is principally based on self-diffusion in which charged polyelectrolyte chains are adsorbed onto an oppositely charged surface and then rearranged owing to the electrostatic interaction. As the presence of water molecules in the assemblies screens the electrostatic attraction, the surface coverage of polyelectrolyte chains is restricted and the weakly adsorbed chains are more produced. In the spin SA process, however, the adsorption and rearrangement of polyelectrolyte chains on the surface and the elimination of weakly bound chains from the substrate are almost simultaneously achieved by high spinning speed for a short time. Fast elimination rate of water by the spinning process significantly increases the concentration of polyelectrolyte solution during the short deposition time. It also improves the electrostatic attraction between oppositely charged polymers. As a result, the adsorption of polyelectrolyte chains is more enhanced and the thicker layers are obtained although the thin layer is favoured by the centrifugal force and air shear force. Surface planarization of the multilayer films is also achieved because the weakly adsorbed chains are easily eliminated by the air shear force.

To understand quantitatively the formation of multilayers in the spinning process, the effects of polyelectrolyte concentration, spinning speed and amount of added ionic salt were investigated. Fig. 3(a) shows that the absorbance increases linearly with the number of bilayers for all concentrations of polymer solution. The slope indicates the deposited amount of polyelectrolytes after the alternate spin self-assembly of PAH and PSS. This amount is clearly dependent on the concentration of polymer solution, but is not affected per bilayer increases from $5 \AA$ to $40 \AA$ and shows the same dependence on the solution concentration. It may be due to the saturation of polyelectrolytes on the surface. Although the increase of solution concentration tends to produce a thicker adsorbed polymer layer due to the adsorption driving forces such as viscous and electrostatic forces, the excess adsorbed layer with weak binding sites are readily eliminated by the desorption driving forces such as
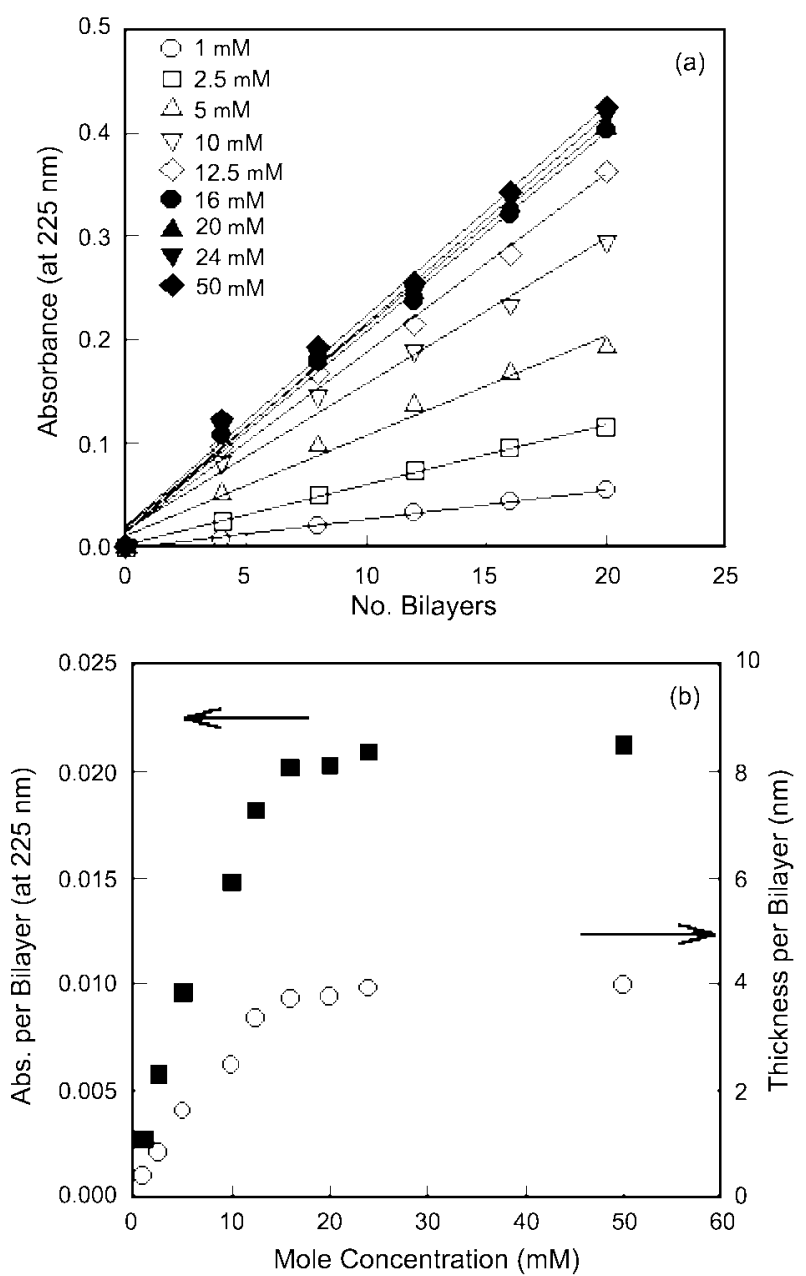

Fig. 3. (a) The increase of absorbance of (PAH/PSS) ${ }_{n}$ as a function of bilayer number. (b) The effect of mole concentration of polyelectrolytes on the adsorbed amount $(O)$ and the thickness per PAH/PSS bilayer $(O)$ prepared by spin SA method. Spinning speed is fixed at 4,000 rpm. 
centrifugal and air shear forces. This observation suggests that it is possible to precisely control the adsorbed amount of polyelectrolytes in multilayer films by simply changing a wide range of solution concentration in spite of the strong repulsion among polyelectrolyte chains with the same charge.

According to theoretical models and experimental results based on the spin coating of single thick layer films, the film thickness $(\mathrm{H})$ is dependent on the spinning speed $(\Omega)$ and the initial solution concentration $\left(\mathrm{C}_{i}\right)$ as follows [Meyerhofer, 1978; Lawrence, 1988; Ohara, 1989; Flack, 1984]:

$$
\mathrm{H} \sim \Omega^{-\alpha} \mathrm{C}_{i}^{\beta}
$$

When the thickness varies from micrometer to millimetre, the value of $\alpha$ is 0.5 and $\beta$ has a value between 1.50 and 2.47. As mentioned above, the absorbance (A) is linearly proportional to the film thickness. Thus, by substituting A for $\mathrm{H}$, the Eq. (1) is examined for the spin SA multilayer thin films. At a fixed solution concentration, the absorbance per bilayer decreases linearly with the spin speed as
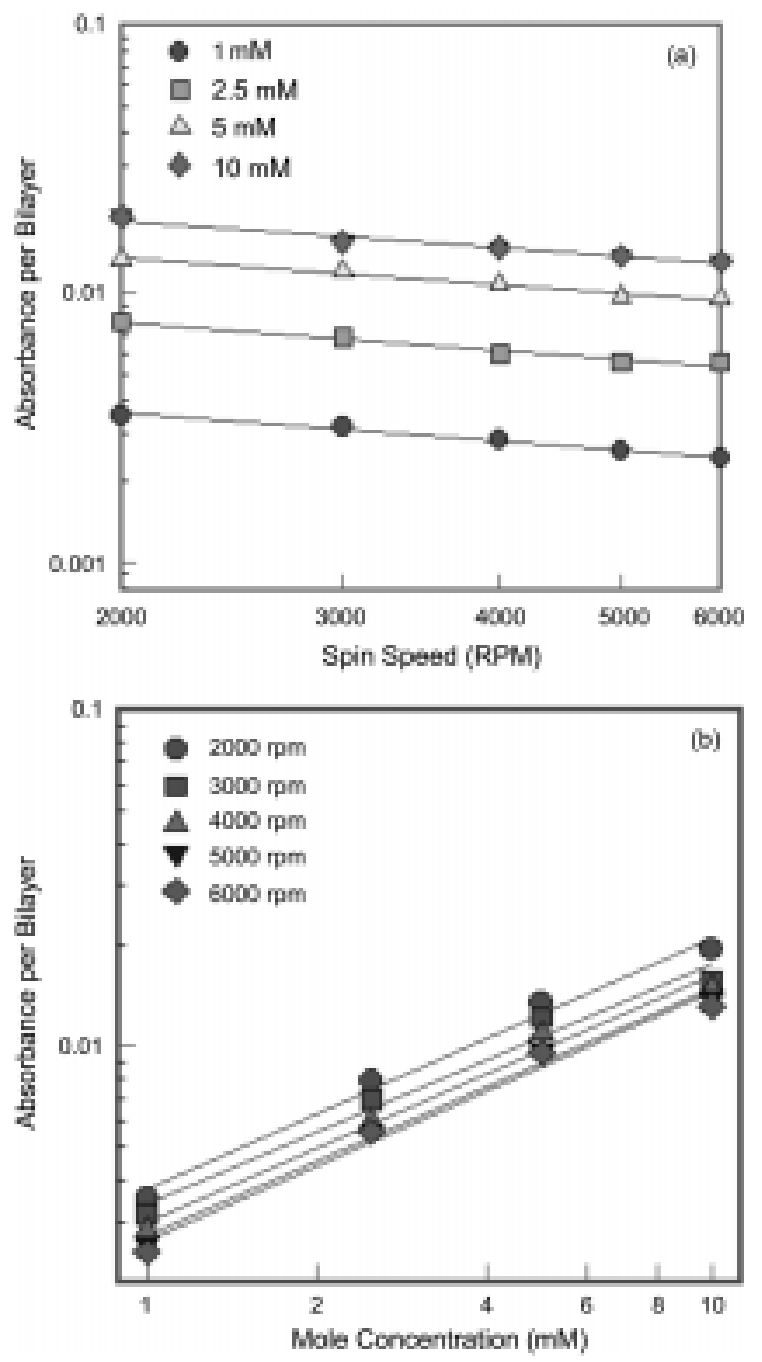

Fig. 4. The effect of spinning speed (a) and mole concentration of polyelectrolytes (b) ranging from $1 \mathrm{mM}$ to $10 \mathrm{mM}$ on the adsorbed amount of PAH/PSS bilayers prepared by the spin SA method. Each point in the figure represents the average absorbance per bilayer for up to 20 bilayers. shown in Fig. 4(a) and the exponent of $\alpha$ is found to be 0.34 . The polyelectrolyte concentrations are used only below $16 \mathrm{mM}$, above which the surface is fully saturated and is not affected by the concentration as confirmed in Fig. 3. Fig. 4(b) represents a log-log plot of the average absorbance per bilayer as a function of mole concentration. For spinning speeds ranging from 2,000 to 6,000 rpm, the absorbance increases with the polyelectrolyte concentration at a scaling exponent of 0.74 . These differences in the power exponents with respect to both the spinning speed and the initial solution concentration may be caused by the characteristics of the spin SA method. In the spin self-assembled multilayer thin films, the weakly bound chains are thoroughly eliminated by the centrifugal force and air shear force during the washing step. As a result, a stable and ultrathin layer is obtained at each deposition step. It means that the next adsorption becomes strongly dependent on the interaction between a deposited sublayer (or a substrate) and a coating overlayer. Compared with a thick single layer, the adsorption and desorption forces, which are determined by only the spinning speed and mole concentration, become less important.

The effect of $\mathrm{NaCl}$ electrolyte concentration on the thickness of multilayer film is represented in Fig. 5. As the ionic strength of PSS solution is increased by the addition of $\mathrm{NaCl}$ salt, the adsorbed

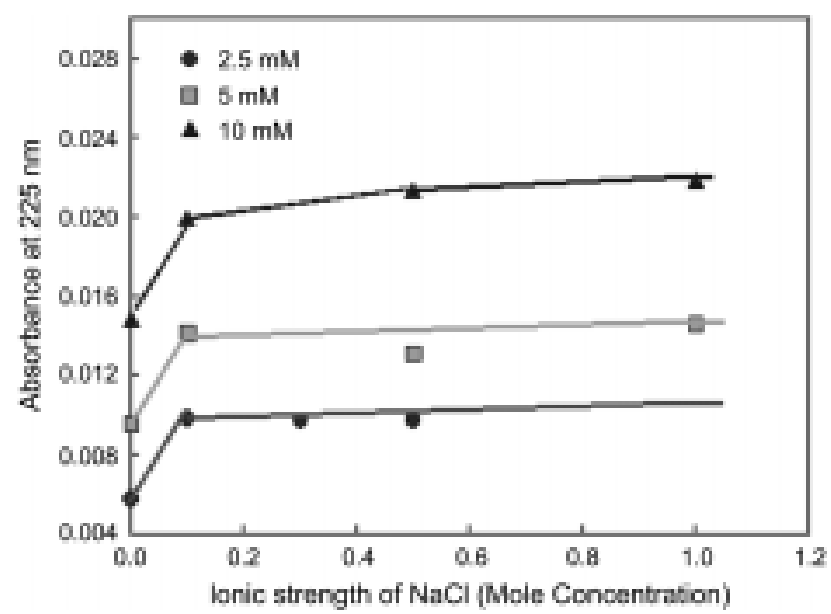

Fig. 5. The effect of ionic strength on the absorbance for various polyelectrolyte concentration.

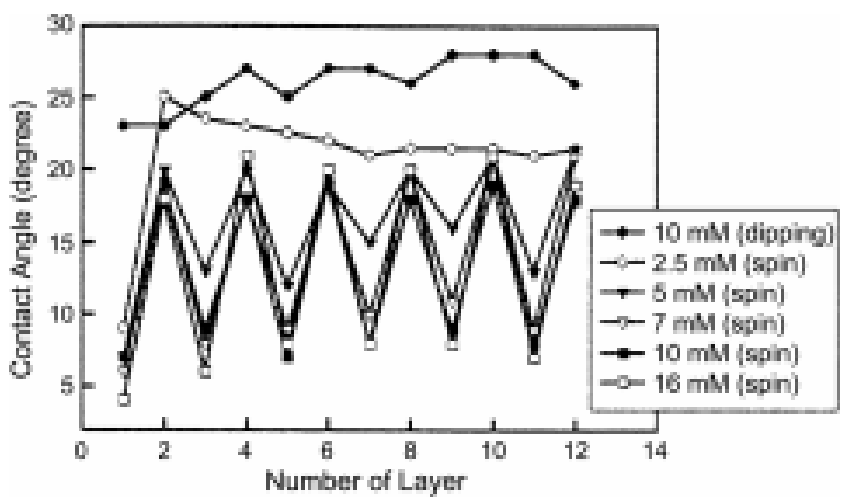

Fig. 6. The contact angles of multilayers prepared by the dipping SA method and the spin SA method with increasing number of layers. 
amount and the thickness of polyelectrolyte films are increased. For various concentrations of polyelectrolyte solution, the absorbance increases up to only $0.1 \mathrm{mM}$, which is lower than the concentration observed in dipping SA method, 0.5 mM [Cho et al., 2000].

To investigate the regularity and surface roughness of multilayer films, the contact angle was measured as shown in Fig. 6. The contact angles of multilayers prepared by the dipping SA method are not affected by the alternate deposition of polyelectrolytes. On the contrary, zigzag behavior of contact angles is observed in the multilayers prepared by the spin SA method. It clearly indicates that the surface property is dominated by the only polyelectrolyte that is deposited at each alternate step. The conventional dipping SA method utilizes a self-diffusion process of charged species onto an oppositely charge surface in a dilute dipping solution. Thus a fuzzy internal structure is produced by the interpenetration between oppositely charged layers. As a result, the contact angle at each layer is not determined by the deposited polyelectrolyte and becomes larger due to the increased surface roughness. When prepared with the spin process, the multilayer film contains rather distinct interfaces between respective layers and the surface is also very smooth. Thus, as the solution concentration increases, the contact angle at each layer approaches more closely to the inherent values of polyelectrolytes (for PAH, $\cos \theta=2.6^{\circ} \mathrm{C}$ and for PSS, $\cos \theta=22.3^{\circ} \mathrm{C}$ ). But, at the low concentration $(2.5 \mathrm{mM})$, the contact angle does not show any regularity because the surface coverage is not enough.

In the self-assembled multilayer films prepared with PPV and polyanion, the polyanion influences the amount of conjugated PPV deposited per bilayer, the thickness of each layer and the electrical properties of the films. To improve device performance, PMA and PSS are inserted in the multilayers as hole blocking layer and hole transporting layer, respectively. The current-voltage-light (I-V-L) characteristics were measured for multilayer electroluminescence (EL) devices prepared by the conventional dipping method and the spin SA method. Fig. 7 shows the relative comparison of the device efficiency, which is the slope of L-I curve. (PPV/PMA) ${ }_{20}$ multilayers show higher efficiency of EL device than (PPV/PSS) $)_{20}$ multilay-

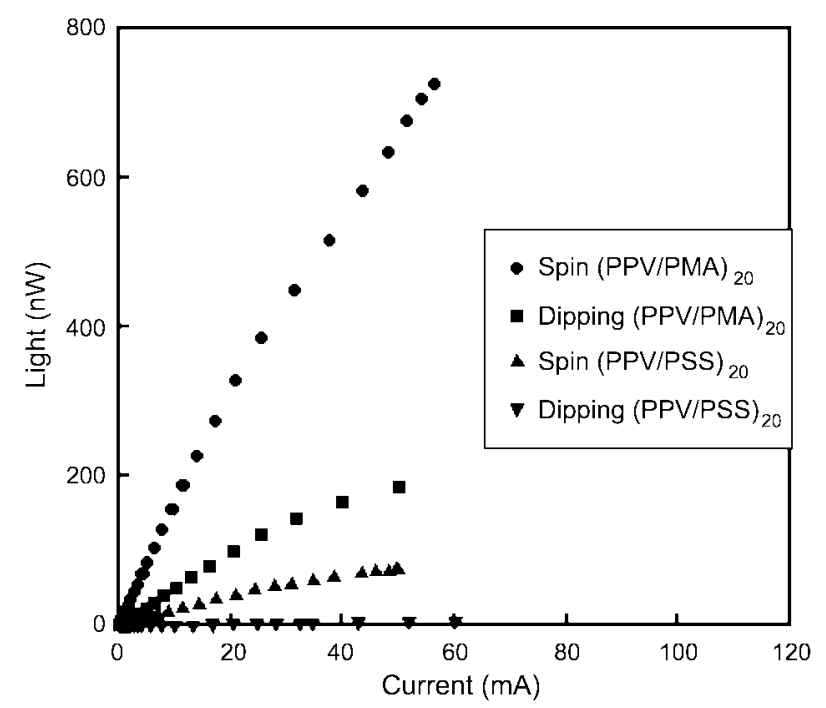

Fig. 7. Relative device efficiency of (PPV/PMA $)_{20}$ and (PPV/PPS $)_{20}$ multilayers prepared by the dipping SA method and the spin SA method. ers because the quantum efficiency of EL devices is dependent on the balanced recombination of holes and electrons. For both multilayers, the spin self-assembled devices show higher quantum efficiency than the dipping self-assembled devices. The reason is that the spin SA method controls precisely both the bilayer thickness and the adsorption amount and also produces the distinct interface. On the contrary, the dipping self-assembled device has a fuzzy internal structure and thus the recombination of holes and electrons is less enhanced.

\section{CONCLUSIONS}

In summary, we have demonstrated for the first time that the spin SA process utilizing centrifugal force, viscous force, air shear force and electrostatic interactions causes the adsorption, the rearrangement of polymer chains onto a substrate and the desorption of weakly bound chains in a very short time of approximately 10 seconds. This new ultrathin film-forming process yields a highly ordered internal structure far superior to the structure obtained by the conventional dipping SA process although it is much simpler and faster. It also allows us to precisely control and predict the bilayer thickness as well as the surface roughness. Multilayer films employing hydrogen bonding and chemical bonding as well as electrostatic attraction can also be fabricated by the spin SA method. In the light of wide application spectra of the ultrathin multilayer films, the spin SA process developed in the present study opens up new possibilities for highly efficient electronic/photonic devices based on multilayer structures.

\section{ACKNOWLEDGMENTS}

This work was financially supported by the National Research Laboratory Program (Grant M1-0104-00-0191) and funded in part by the Ministry of Education through the Brain Korea 21 Program at Seoul National University.

\section{REFERENCES}

Chou, F. C. and Wu, P.-Y., "Effect of Air Shear on Film Planarization During Spin Coating,' J. Electrochem. Soc., 147, 699 (2000).

Cho, J., Char, K., Kim, S. Y., Hong, J.-D., Lee, S. K. and Kim, D. Y., "Effect of Added Ionic Salt on the Quantum Efficiency of Self-assembled Films Prepared with Poly(p-phenylene vinylene),' Thin Solid Films, 379, 188 (2000).

Decher, G., "Fuzzy Nanoassemblies: Toward Layered Polymeric Multicomposites,' Science, 277, 1232 (1997).

Flack, W. W., Soong, D. S., Bell, A. T. and Hess, D. W., "A Mathematical Model for Spin Coating of Polymer Resists,' J. Appl. Phys., 56, 1199 (1984).

He, Y., Gong, S., Hattori, R. and Kanicki, J., "High Performance Organic Polymer Light-Emitting Heterostructure Devices,' Appl. Phys. Lett., 74, 2265 (1999).

Ho, P. K. H., Kim, J. S., Burroughes, J. H., Becker, H., Li, S. F. Y., Brown, T. M., Cacialli, F. and Friend, R. H., "Molecular-scale Interface Engineering for Polymer Light-Emitting Diodes,' Nature, 404, 481 (2000).

Ho, P. K. H., Granstrom, M., Friend, R. H. and Greenham, N. C., 
"Ultrathin Self-Assembled Layers at the ITO Interface to Control Charge Injection and Electroluminescence Efficiency in Polymer Light-Emitting Diodes,' Adv. Mater., 10, 769 (1998).

Hong, H., Steitz, R., Kirstein, S. and Davidov, D., "Superlattice Structures in Poly(phenylene vinylene)-Based Self-Assembled Films,' Adv. Mater., 10, 1104 (1998).

Kim, J. S., Nam, T. Y. and Huh, Y. J., "The Optical Characteristics in the Layers of Compact Disc-Recordable,' Korean J. Chem. Eng., 14, 88 (1997).

Kotov, N., Dekang, I. and Fendler, J. H., "Layer-by-layer Self-Assembly of Polyelectrolyte-Semiconductor Nanoparticle Composite Films," J. Phys. Chem., 99, 13065 (1995).

Laschewasky, Wischerhoff, E., Kauranen, M. and Persoons, A., "Polyelectrolyte Multilayer Assemblies Containing Nonlinear Optical Dyes,' Macromolecules, 30, 8304 (1997).

Lawrence, C. J., 'The Mechanics of Spin Coating of Polymer Films,' Phys. Fluids, 31, 2786 (1988).

Lenahan, K. M., Wang, Y. X., Liu, Y. J., Claus, R. O., Heflin, J. R., Marciu, D. and Figura, C., "Novel Polymer Dyes for Nonlinear Optical Applications Using Ionic Self-Assembled Monolayer Technology,' Adv. Mater., 10, 853 (1998).
Lvov, Y., Ariga, K., Ichinose, I. and Kunitake, T., “Assembly of Multicomponent Protein Films by Means of Electrostatic Layer-by-layer Adsorption,' J. Am. Chem. Soc., 117, 6117 (1995).

Mendelson, J. D., Barrett, C. J., Chan, V. V., Pal, A. J., Mayes, A. M. and Rubner, M. F., "Fabrication of Microporous Thin Films from Polyelectrolyte Multilayers,' Langmuir, 16, 5017 (2000).

Meyerhofer, D., "Characteristics of Resist Films Produced by Spinning,' J. Appl. Phys., 49, 3973 (1978).

Ohara, T., Matsumoto, Y. and Ohasi, H., "The Film Formation Dynamics in Spin Coating,' Phys. Fluids A, 1, 1949 (1989).

Onitsuka, O., Fou, A. C., Ferreira, M., Hsieh, B. R. and Rubner, M. F., "Enhancement of Lightemitting Diodes Based on Self-assembled Heterostructures of Poly(p-phenylene vinylene),' J. Appl. Phys., 80, 4067 (1996).

Park, Y. O., Park, H. S., Park, S. J., Kim, S. D., Choi, H. K. and Lim, J. H., "Development and Evaluation of Multilayer Air Filter Media,' Korean J. Chem. Eng., 18, 1020 (2001).

Zhang, X. and Shen, J., "Self-assembled Ultrathin Films from Layered Nanoarchitectures to Functional Assemblies," Adv. Mater., 11, 1139 (1999). 